\title{
Estructura del ensamblaje de macroinvertebrados bentónicos de una laguna urbana en la Pampa Mesopotámica
}

\section{Structure of the benthonic macroinvertebrate assemblage of an urban lagoon in the Mesopotamic Pampa}

\author{
Melina Crettaz-Minaglia ${ }^{1,2 *}$,Diamela Gianello ${ }^{1,3}$, Carlos Roldán $\odot^{1}$, Eduardo Chaves ${ }^{1}$, Irene Aguer ${ }^{1}$ y Ricardo A. Juárez®1 \\ *Autor de correspondencia: crettaz.melina@uader.edu.ar
}

Recibido: 2 de mayo de 2018 - Aceptado: 18 de septiembre de 2018

1 Laboratorio de Indicadores Biológicos y Gestión Ambiental de Calidad de Agua, Facultad de Ciencia y Tecnología, UADER, Gualeguaychú, Entre Ríos, Argentina.
2 Área de Biología y Bioinformática, Instituto de Ciencias, UNGS, Gualeguaychú, Entre Ríos, Argentina.
3 Grupo de Ecología de Sistemas Acuáticos a escala de Paisaje, Instituto de Investigaciones en Biodiversidad y Medio Ambiente, UNComahue-CONICET, Río Negro, Argentina.

\begin{abstract}
Resumen
Palabras clave:

diversidad; Entre Ríos; laguna urbana; macroinvertebrados

El objetivo de este trabajo fue estudiar la estructura del ensamblaje de macroinvertebrados bentónicos de la laguna del Parque Unzué y discutir sobre su calidad de agua. El área de estudio se encuentra en la ciudad de Gualeguaychú (Entre Ríos, Argentina), sobre la margen izquierda del río Gualeguaychú. La laguna tiene un área de $2750 \mathrm{~m}^{2}$ y una profundidad máxima de 1,70 m. Durante 2016-2017, se muestreó en 3 puntos $(\mathrm{n}=10)$, se midieron parámetros in situ y se recolectaron muestras del bentos, se caracterizaron los sedimentos y los macroinvertebrados se identificaron hasta el menor nivel taxonómico posible, y se los agrupó en grupos funcionales alimenticios (GFA). Se calculó la diversidad, la diversidad máxima esperada (Hmáx) y los números de Hill. No se hallaron diferencias estadísticamente significativas $(\mathrm{p}<0,05)$ entre los puntos de muestreo y los parámetros in situ; sin embargo, respecto a los macroinvertebrados, los puntos 1 y 3 presentaron mayor similitud debido a la composición en el sustrato. Se recolectaron 1726 individuos distribuidos en 13 taxones: Oligochaeta (1), Hirudinea (1), Bivalvia (4), Gastropoda (3), Amphipoda (1) e Insecta (3), característicos de ambientes con abundante materia orgánica. La diversidad fue baja y coincidió con la Hmáx solo en el $23 \%$ de los casos, el número de especies efectivas varió de uno a cinco y los taxones dominantes representaron el 40 \% de la riqueza. Respecto a los GFA, la abundancia se distribuyó en: colector-recolector, colector-filtrador y depredador y, la biomasa, en el raspador y el colector-filtrador. La laguna del Parque Unzué evidenció efectos de contaminación orgánica durante todo el período de muestreo reflejado en la estructura y composición del ensamblaje de macroinvertebrados.
\end{abstract}

Key words:

diversity; Entre Ríos; urban lagoon; macroinvertebrates

\begin{abstract}
The aim of this work was to study the structure of the benthonic macroinvertebrate assemblages in the Parque Unzué lagoon and to discuss about its water quality. The area of study is located in Gualeguaychú city (Entre Ríos, Argentina), on the left margin from Gualeguaychú river. The lagoon has an area of 2750 $\mathrm{m}^{2}$ and a maximum depth of $1.70 \mathrm{~m}$. During 2016-2017, some samples were taken at 3 points $(\mathrm{n}=10)$, in situ parameters were measured and benthic samples were collected, sediments were characterized and macroinvertebrates were identified into the lowest possible taxonomic level and grouped into functional groups food (GFA). Richness (S) and diversity were calculated and the macroinvertebrates were classified into functional feeding group (FFG). No significant differences were found $(\mathrm{p}<0.05)$ between sample sites and in situ parameters, however, with respect to macroinvertebrates, the sample sites 1 and 3 were more similar than those with site 2. Diversity, maximum diversity (Hmax) and Hill's numbers were calculated. No statistically significant differences $(\mathrm{p}<0.05)$ were found between the sampling points and the in situ parameters; however, with respect to macroinvertebrates, points 1 and 3 presented greater similarity than these with point 2, possibly due to differences in the substrate. 1726 individuals distributed in 13 taxa: Oligochaeta (1), Hirudinea (1), Bivalvia (4), Gastropoda (3), Amphipoda (1), and Insecta (3). All of these are characteristic of large amounts of organic matter environments. Diversity was low, this coincided with maximum diversity on $23 \%$ of the cases, and the dominant taxa represent $40 \%$ of richness. The FFG abundance was distributed in gathering-collectors, filtering-collectors and predators and, the biomass, in scrapers, and filtering-collectors. The lagoon from Parque Unzué showed effects of organic pollution during the whole sampling period reflected in the structure and composition of the macroinvertebrate assemblage.
\end{abstract}




\section{Introducción}

Las lagunas urbanas son ecosistemas presentes en muchas ciudades y forman parte de la ecología urbana (Mancini et al., 2012). Estas han sido construidas para diversos fines; proporcionan hábitats que permiten el establecimiento de diferentes especies, tanto acuáticas como terrestres, migratorias o residentes, e incrementan su valor paisajístico (García-Rodríguez et al., 2014) y mitigan las adversidades del clima urbano (Sorensen et al., 1998). A su vez, son utilizadas para la realización de actividades educacionales y recreativas (Quirós, 2007; Mancini et al., 2012). Estos ambientes se encuentran dentro de los sitios RAMSAR como humedales continentales; por ello, ante la pérdida de humedales naturales en la región pampeana argentina, existe una necesidad creciente de considerar estos ambientes urbanos como sitios susceptibles de ser protegidos, por lo que es fundamental conocer la biodiversidad que albergan (Taborda et al., 2017). Estos ecosistemas suelen estar afectados por la fragmentación del hábitat y la contaminación orgánica (Sorensen et al., 1998; Elosegui y Sabater, 2009) y esto repercute en la composición y distribución de los ensamblajes de especies que, a su vez, pueden considerarse como indicadores de salud urbana (Hough, 1998). La magnitud de los cambios en los ensamblajes depende del tiempo que dure la perturbación en el sistema acuático, su intensidad y su naturaleza (Ospina y Peña, 2004). Debido a esto, los ensamblajes de especies se utilizan ampliamente para determinar la calidad del agua (Montoya et al., 2011) analizando, entre otros, la densidad de especies, diversidad específica, aspectos alimenticios y flujo de energía (Silva et al., 2009).

En particular, el grupo de macroinvertebrados bentónicos está compuesto por organismos de tamaño $<500 \mu \mathrm{m}$ que incluyen moluscos, insectos, oligoquetos y nematodos; habitan en los sedimentos de los cursos de agua y son nexo entre los consumidores en las redes tróficas (Barbour et al., 1999). Además, son capaces de reflejar diferentes perturbaciones antrópicas como contaminación orgánica, acidez y pérdida de hábitats, a través de cambios en su estructura (Plafkin et al., 1989; Pavé y Marchese, 2005). También presentan distintas especializaciones asociadas a la adquisición de alimento, que permiten clasificarlos en grupos funcionales o gremios tróficos, para poder explotar una variedad de recursos (Merritt y Cummins, 1978; Cummins y Klug, 1979; Velázquez y Miserendino, 2003).

Las ciudades latinoamericanas y, en general, las ciudades alrededor del mundo, han dado muy poco peso a la planeación del territorio al estudio de las lagunas urbanas 113 como un asunto urgente y relevante; a pesar de las pérdidas naturales diarias en materia de biodiversidad y las premisas por la sustentabilidad global y regional, en un mundo con una tendencia creciente a lo urbano (Sierra-Vásquez, 2012). Si bien Argentina tiene una diversidad importante de lagunas urbanas, los estudios se han centrado principalmente en aspectos epidemiológicos $y$, en particular, los estudios de macroinvertebrados bentónicos en estos ambientes son escasos (Faggi y Martínez-Carretero, 2013). Estos autores mencionan que se cuenta con abundante bibliografía sobre mosquitos de las ciudades de Buenos Aires, Santa Fe y Santo Tomé que se ha enfocado en conocer las variables climáticas y los hábitats disponibles que regulan la abundancia de las larvas, con el fin de encarar programas de monitoreo y control sanitario eficaces. El objetivo de este trabajo fue estudiar la estructura de los ensamblajes de macroinvertebrados bentónicos de la laguna del Parque Unzué y discutir sobre su calidad de agua.

\section{Materiales y Métodos}

\section{Área de estudio}

El área de estudio se encuentra dentro del tejido de la ciudad turística de Gualeguaychú, en la Pampa Mesopotámica (Morello et al., 2012); es uno de los centros poblados más grandes de la provincia de Entre Ríos que concentra 83116 habitantes (INDEC, 2010). Frente a la ciudad, situado sobre la margen izquierda del río Gualeguaychú, se encuentra una importante área recreativa (Gianello et al., 2017) denominada Parque Unzué, el cual cuenta con una laguna artificial $\left(33^{\circ} 00^{\prime} 46,30^{\prime \prime} \mathrm{S}, 58^{\circ} 29^{\prime} 23,41^{\prime \prime} 0\right)$. Esta tiene una profundidad de $1,7 \mathrm{~m}$, un perímetro de 0,82 $\mathrm{km}$, un área de $2,75 \mathrm{~km}^{2}$, una longitud máxima de 0,32 $\mathrm{km}$ y un ancho máximo de $0,14 \mathrm{~km}$. Su uso es recreativo sin contacto directo (Gianello, 2016; Taborda et al., 2017) y como sumidero de biodiversidad. Sus aportes hídricos provienen de las precipitaciones o eventuales desbordes del río Gualeguaychú (Gianello et al., 2017) y recambios para compensar las pérdidas del nivel de agua. Asimismo, durante el verano suele producirse un aporte puntual de efluentes cloacales provenientes del camping municipal lindero cuando se produce la sobrecarga del sistema de tratamiento.

Presenta siete especies de flora nativa y seis de flora exótica, y exhibe alteraciones de las riberas por presencia de caminos y laderas de hormigón. Respecto al fitoplancton, en la laguna se han hallado treinta géneros, representantes de Bacillariophyceae (11), Chlorophyta (10), Euglenophyta (4), Cyanobacteria (4) y Dinophyceae (1) (Gianello et al., 2017). En cuanto a la fauna, cuenta particularmente 
con una importante diversidad de aves (34 especies), incluyendo especies migratorias como la garcita blanca (Egretta thula), ave migratoria de América, que frecuenta la laguna durante el período estival del hemisferio sur, seis especies de peces, dos de reptiles, uno de mamíferos autóctonos y dos exóticos. (Gianello, 2016; Taborda et al., 2017).

\section{Puntos y frecuencia de muestreo}

Con el fin de describir las características de la laguna, se seleccionaron tres puntos (figura 1) donde se realizaron 10 muestreos entre mayo de 2016 y diciembre de 2017, abarcando todas las estaciones climáticas. La frecuencia de los muestreos fue propuesta cada dos meses; sin embargo, esta estuvo sujeta a las condiciones climáticas y de acceso al sitio de estudio.

\section{Medición de parámetros in situ}

En cada punto de muestreo, se midió la profundidad y los siguientes parámetros: $\mathrm{pH}$, temperatura, conductividad eléctrica (CE) y sólidos disueltos totales (SDT) con medidor multiparamétricos Hanna HI991003. Además, se midió la temperatura del aire con termómetro de mercurio y oxígeno disuelto (OD) con medidor YSI modelo 55. Todos los equipos fueron previamente calibrados (APHA-AWWAWFF, 2013).

Adicionalmente, se registraron las precipitaciones provistas por la Dirección de Hidráulica de Entre Ríos y, teniendo en cuenta que en ocasiones la laguna recibe aportes debido a desbordes del río y que los recambios se realizan con agua de este, se registró la altura del río Gualeguaychú (Prefectura Naval Argentina).

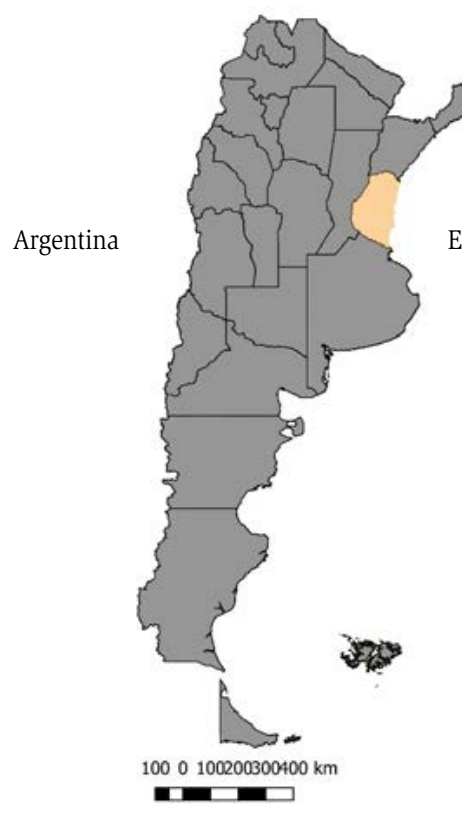

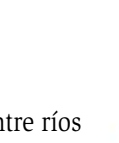

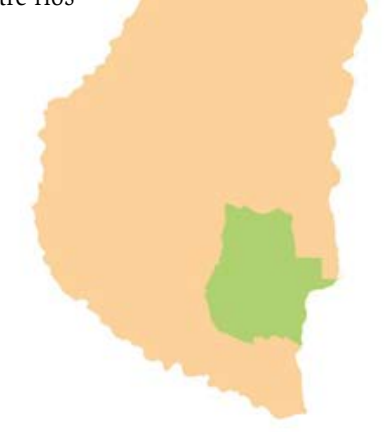

$25 \quad 0 \quad 25 \quad 50 \quad 75100 \mathrm{~km}$

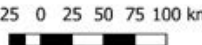

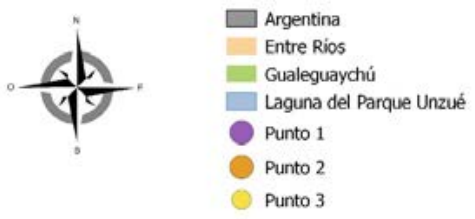

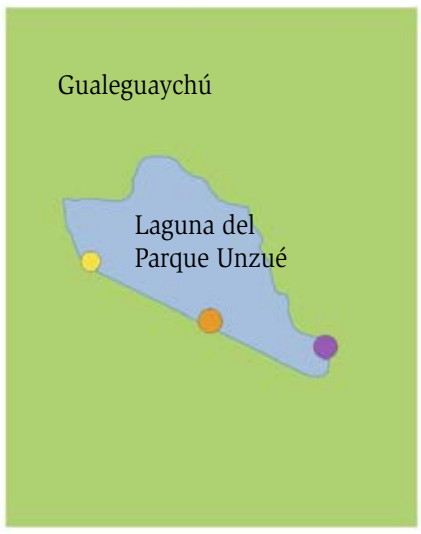

$250255075100 \mathrm{~m}$

$255075100 \mathrm{~m}$

Figura 1. Área de estudio (QGIS, 2018).

\section{Muestreo de macroinvertebrados bentónicos}

Los macroinvertebrados bentónicos fueron muestreados siguiendo los criterios de Darrigran et al. (2007), utilizando una draga Ekman con una superficie de 225 $\mathrm{cm}^{2}$ y volumen máximo de $3,375 \mathrm{~cm}^{3}$. Las muestras se conservaron en campo con solución de formalina al $4 \%$ (Barbour et al., 1999) y fueron refrigeradas hasta su arribo al laboratorio.
En el laboratorio, las muestras se lavaron y se filtraron con un tamiz de $200 \mu \mathrm{m}$ de poro. Luego se realizó la tinción de los componentes orgánicos con Eritrocina B en una dilución del 0,4 \% en volumen (Rodrígues-Capítulo et al., 2010), para facilitar la separación de los organismos. Las muestras se observaron con una lupa estereoscópica Motic con aumentos de $10 \mathrm{X}$ y $30 \mathrm{X}$, se cuantificaron organismos completos y se identificaron hasta el menor nivel taxonómico posible (Barbour et al., 1999; Prat et al., 
2009), utilizando las claves taxonómicas de Lopretto y Tell (1995) y Domínguez y Fernández (2009). Para obtener la biomasa húmeda, cada individuo fue pesado directamente con balanza analítica Ohaus modelo PA 224C (precisión $=0,1 \mathrm{mg}$ ). Además, se realizó una valoración cuantitativa de los sedimentos extraídos, a los cuales se le tomaron las dimensiones (Juárez et al., 2016).

\section{Análisis de datos}

Los datos obtenidos fueron analizados estadísticamente utilizando estadísticos paramétricos, previo análisis del cumplimiento de los supuestos paramétricos (Hammer et al., 2001) utilizando para ello el programa PAST (Paleontological Statistic; Hammer et al., 2001), Microsoft Excel ${ }^{\circledR}$ y Systat Software Inc ${ }^{\circledR}$ versión 12.0. Para conocer la homogeneidad del área de estudio, se aplicó un ANOVA paramétrico de una vía para detectar diferencias significativas $(\mathrm{p}<0,05)$ entre los puntos de muestreo (Villanueva y Chamané-Zapata, 2016) para los parámetros in situ con el programa PAST. Con el mismo objetivo, se compararon los taxones presentes en cada punto de muestreo mediante el análisis de agrupamiento de Bray-Curtis (1957) (Ferreira-Rezendo, 2007).

Se calculó la riqueza (S), abundancia y biomasa húmeda de los taxones. Se aplicó el índice de diversidad de ShannonWeaver (1949) $\left(\mathrm{H}^{\prime}\right)$ como reflejo de la heterogeneidad del ensamblaje (Pla, 2006), también se calculó la diversidad máxima esperada como H'max = lnS, la cual es alcanzada cuando todos los taxones están igualmente presentes (Pla, 2006). Además, se calculó el antilogaritmo de $\mathrm{H}^{\prime}$ como
$\mathrm{e}^{\mathrm{H}}$ ' que indica el número de especies efectivas, es decir, el número de especies equiabundantes necesarias para alcanzar $\mathrm{H}^{\prime}$. Cuanto mayor sea la diferencia entre $\mathrm{e}^{\mathrm{H}^{\prime}}$ y S, menos diverso será el ensamblaje (Pla, 2006). Por otro lado, se aplicó el índice de dominancia de Simpson (D) y se calculó 1/D como indicador del número de taxones dominantes. Estos cálculos responden a los números de diversidad de Hill (1973) dónde $\mathrm{N} 0=\mathrm{S} ; \mathrm{N} 1=\mathrm{e}^{\mathrm{H}^{\prime}}$ y N2 = 1/D.

Los macroinvertebrados fueron clasificados según los grupos funcionales alimenticios (GFA) propuestos por Cummins et al. (2005), Rodríguez-Barrios et al. (2011), Andramunio-Acero y Caraballo (2012) y Ocón et al. (2013) y se determinó la biomasa de cada GFA a partir de los datos obtenidos de la biomasa de cada individuo. Los datos de tamaño de sedimento fueron clasificados de acuerdo con Barbour et al. (1999).

\section{Resultados}

\section{Parámetros in situ, precipitaciones y altura del río}

Los estadísticos descriptivos de los parámetros determinados in situ, los registros de precipitaciones y altura del río Gualeguaychú pueden observarse en la tabla 1. De acuerdo con el análisis de ANOVA, no se hallaron diferencias significativas $(\mathrm{p}>0,05)$ entre los puntos de muestreo respecto a los parámetros in situ. Con lo anterior se observó que el área de estudio fue relativamente homogénea desde el punto de vista espacial.

Tabla 1. Estadísticos descriptivos de variables ambientales: $\overline{\mathrm{x}}=$ promedio, $\mathrm{m}=$ valor mínimo, $\mathrm{M}=$ valor máximo, $\mathrm{ES}=$ error estándar. Variables ambientales: T. Aire = temperatura del aire, T. Agua = temperatura del agua, $\mathrm{CE}=$ conductividad eléctrica, $\mathrm{SDT}=$ sólidos disueltos totales, $\mathrm{OD}=$ oxígeno disuelto, Prof. $=$ profundidad, $\mathrm{PSPM}=$ precipitaciones semana previa al muestreo, $\mathrm{PMPM}=$ precipitaciones mes previo al muestreo.

\begin{tabular}{|c|c|c|c|c|c|c|c|c|c|c|c|c|}
\hline & \multicolumn{4}{|c|}{ Punto 1} & \multicolumn{4}{|c|}{ Punto 2} & \multicolumn{4}{|c|}{ Punto 3} \\
\hline & $\overline{\mathbf{x}}$ & m & M & ES & $\overline{\mathbf{x}}$ & m & M & ES & $\overline{\mathbf{x}}$ & $\mathbf{M}$ & M & ES \\
\hline T. Aire $\left({ }^{\circ} \mathrm{C}\right)$ & 18,90 & 10,00 & 28,00 & 1,92 & 19,80 & 11,00 & 30,00 & 2,24 & 20,50 & 12,00 & 32,00 & 2,37 \\
\hline T. Agua $\left({ }^{\circ} \mathrm{C}\right)$ & 19,80 & 13,00 & 28,50 & 1,92 & 20,50 & 12,70 & 28,80 & 1,88 & 20,50 & 13,00 & 30,00 & 1,96 \\
\hline $\mathrm{pH}$ & 6,98 & 5,75 & 8,04 & 0,20 & 7,34 & 6,05 & 9,05 & 0,28 & 7,23 & 6,04 & 8,90 & 0,24 \\
\hline $\mathrm{CE}\left(\mu \mathrm{S} \mathrm{cm}^{-1}\right)$ & 434,00 & 127,00 & 690,00 & 58,19 & 429,00 & 162,00 & 1160,0 & 92,65 & 393,00 & 210,00 & 540,00 & 43,32 \\
\hline SDT $\left(\mathrm{mg} \mathrm{L}^{-1}\right)$ & 209,00 & 64,00 & 281,00 & 25,93 & 211,00 & 81,00 & 560,00 & 44,90 & 191,00 & 105,00 & 268,00 & 19,61 \\
\hline OD (mg L-1) & 3,96 & 1,68 & 8,90 & 0,76 & 5,63 & 1,55 & 14,30 & 1,34 & 4,48 & 0,95 & 11,50 & 0,98 \\
\hline Prof. (m) & 0,50 & 0,30 & 0,70 & 0,03 & 0,50 & 0,30 & 0,70 & 0,03 & 0,40 & 0,50 & 0,30 & 0,03 \\
\hline PSPM (mm) & 33,08 & 0,00 & 95,00 & 10,02 & 33,08 & 0,00 & 95,00 & 10,02 & 33,08 & 0,00 & 95,00 & 10,02 \\
\hline $\begin{array}{l}\text { PMPM (mm) } \\
\text { Altura del río }\end{array}$ & 132,61 & 16,50 & 359,00 & 28,11 & 132,61 & 16,50 & 359,00 & 28,11 & 132,61 & 16,50 & 359,00 & 28,11 \\
\hline $\begin{array}{l}\text { Gualeguaychu } \\
(\mathrm{cm})\end{array}$ & 1,87 & 1,24 & 2,58 & 0,14 & 1,87 & 1,24 & 2,58 & 0,14 & 1,87 & 1,24 & 2,58 & 0,14 \\
\hline
\end{tabular}


La temperatura del agua y del aire variaron de acuerdo con el régimen climático estacional que caracteriza a la Pampa Mesopotámica, se hallaron los menores valores en otoño-invierno y los mayores, en primaveraverano. La temperatura del aire varió de $10{ }^{\circ} \mathrm{C}$ en el punto $1(14 / 05 / 16)$ a $32{ }^{\circ} \mathrm{C}$ en el punto $3(15 / 11 / 17)$. La temperatura mínima del agua fue de $12,7{ }^{\circ} \mathrm{C}$ y se registró en el punto 2 el 09/07/16 y la máxima, de 30 ${ }^{\circ} \mathrm{C}$ en el punto 3, el 20/01/17. Asimismo, las mayores precipitaciones ocurrieron en primavera-verano y se registraron incrementos en el nivel hidrométrico del río Gualeguaychú en otoño-invierno.

Por otra parte, el pH varió de 5,75 a 9,05, y se halló el valor mínimo en el punto 1 el 08/09/17 y el máximo en el punto 2 el 20/01/17. La CE varió de 127 a 1160 $\mu \mathrm{S} . \mathrm{cm}^{-1}$, y los SDT 60 a 560 mg.L $\mathrm{L}^{-1}$; los valores mínimos de estos dos parámetros se registraron en el punto 1 el 16/03/17 y los máximos en el punto 2 el 09/07/16. En cuanto al OD, el valor mínimo fue de $0,95 \mathrm{mg} \cdot \mathrm{L}^{-1}$ en el punto 3 el 16/03/17 y el máximo de 14,3 en el punto 2 el 10/09/16.

\section{Macroinvertebrados}

Se recolectaron 1726 individuos distribuidos en 13 taxones; 2 órdenes, 4 familias, 4 géneros y 3 especies representantes de los Filo Annelida $(\mathrm{n}=1447)$, Mollusca $(\mathrm{n}=249)$ y Arthropoda $(\mathrm{n}=30)$ (Figura 2). Los puntos 1 y 3 fueron similares respecto a la abundancia de macroinvertebrados (figura 2), representando el 42,2 y $41,5 \%$ de la abundancia $(n=728$ y $n=716)$ respectivamente con 8 taxones presentes; Oligochaeta fue el más abundante (74,0 y 72,4 \%, respectivamente) (figura 2). En el punto 1, además, estuvo presente Pomacea canaliculata $(11,8 \%)$ e Hirudinea $(7,83 \%)$ y, en el punto 3, Hirudinea (17 \%) y Corbicula fluminea, Sphaeriidae, Chironomidae y P. canaliculata (representando el $2 \%$ aproximadamente cada taxón). En el punto 2, a diferencia de los demás, representó el 16,3 \% de la abundancia total de la laguna con 12 taxones presentes; también los más abundante fueron Oligochaeta $(65,2 \%)$, Sphaeriidae $(10,6 \%)$, Hirudinea $(9,57 \%)$ y $P$. canaliculata $(4,61 \%)$ (figura 2).

Respecto a la biomasa, el punto 1 representó el 10,5\% del total, mientras que el punto 2 y 3 representaron el 35,3 y $54,2 \%$. En general, P. canaliculata fue el taxón que mayor biomasa aportó al total de la laguna, seguido de Oligochaeta, $C$. fluminea e Hirudinea. Esta representación varió en el punto 2 dada la presencia de un ejemplar de Mycetopodidae (20/01/2017) (figura 3).

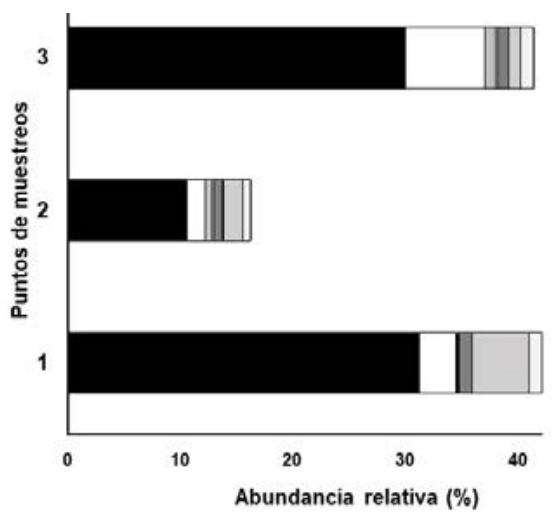

- Oligochaeta $71.90 \%$ 口 Hirudinea $11,93 \%$ = Horvathinia $0,06 \%$ = Corixidae $0,06 \%$ 口Chironomidae $1.51 \%$ H. curvispina $0,12 \%$ 口Ancylidae $0,46 \%$ a Cochliopidae $2,90 \%$ = $P$. canaliculata $0,06 \%$ Mycetopodidae 0,06\% 口Sphaerildae $7.82 \%$ 口 Hyrildae $3.01 \%$ =C. fluminea $0,12 \%$

Figura 2. Abundancia relativa en porcentaje de los taxones presentes en la laguna del Parque Unzué, discriminada por punto de muestreo.

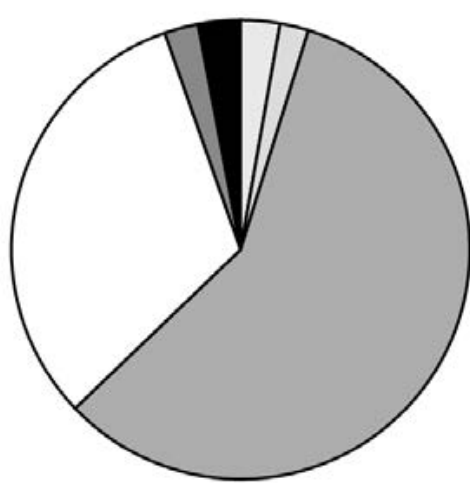

口Oligochaeta $2,83 \% \quad \square$ Hirudinea $2,07 \% \quad \square P$. canaliculata $59,48 \%$ aycetopodidae $32,57 \% \square$ C. fluminea $2,45 \%$ otros $3,05 \%$

Figura 3. Representación de la biomasa húmeda relativa en porcentaje para los taxones presentes en la laguna del Parque Unzué.

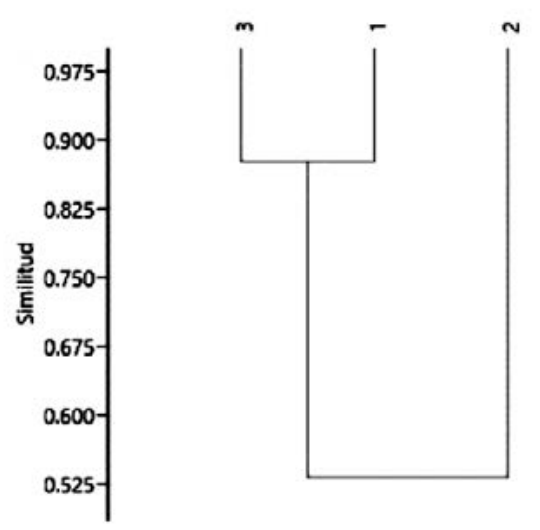

Figura 4. Análisis de agrupamiento basado en las abundancias de macroinvertebrados bentónicos de la laguna del Parque Unzué. $1,2,3$ son los puntos de muestreo. 
El análisis de agrupamiento de Bray-Curtis (figura 4) muestra que los puntos 1 y 3 presentaron mayor similitud que entre estos y el punto 2. En los puntos 1 y 3, de los ocho taxones totales presentes, siete estuvieron con abundancias similares.

En la figura 5, se puede observar la variación de la riqueza y diversidad en cada una de las estaciones de muestreos, así como la diversidad máxima esperada. El S máximo fue de ocho (20/1/2017) y el mínimo de uno (15/11/2017); ambos en el punto 1. En el resto de los puntos de muestreo, $\mathrm{S}$ varió entre siete (20/1/2017) y dos $(29 / 6 / 2017,23 / 12 / 2017)$. La diversidad H' varió de 0,000-1,733, solo en el $23 \%$ de casos H’y Hmáx coincidieron (figura 5). El número efectivo de especies varió de 2,72-15,38 y coincidió con lo registrado en un $30 \%$, la dominancia fue alta en el $20 \%$ de los casos y el número de taxones dominantes fue entre uno y cinco, que corresponden a Oligocheta, Hirudinea, Sphaeriidae, C. fluminea y P. canaliculata (figura 6).

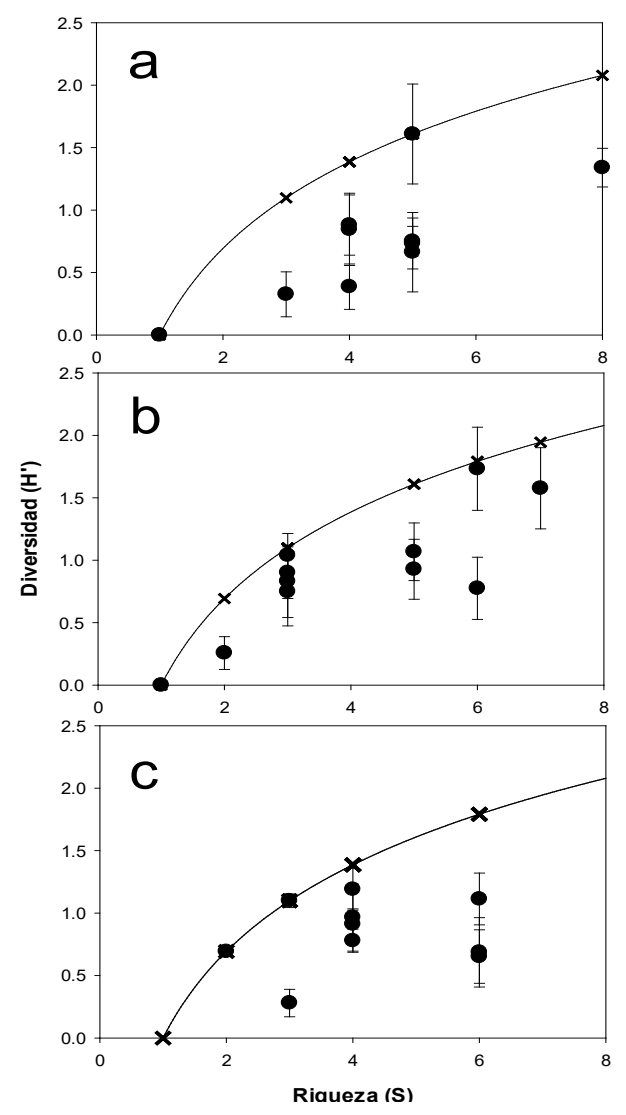

Figura 5. Índice diversidad $\left(\mathrm{H}^{\prime}\right)$ en función del número de taxones (número de Hill N0) para: a) punto 1, b) punto 2, c) punto 3 $(\bullet)$. Además, se graficó la curva logarítmica (x) de la máxima 117 diversidad esperada (H’máx).
Respecto a los GFA (figura 7), los puntos de muestreo evidenciaron similitud $(0,88-0,95)$ y el grupo predominante fue el (CR) $(69,15-75,14 \%)$, representado principalmente por Oligochaeta; seguido de CF (15,25-14,70 \%) en los puntos 1 y 2 , representado por $C$. fluminea y Sphaeriidae; y D $(17,04 \%)$ en el punto 3 , representado por Hirudinea. El grupo raspador por $P$. canaliculata fue menos abundante (2,23-4,96 \%) y el grupo fragmentador solo estuvo presente en el punto 2 y fue representado por $H$. curvispina $(0,71$ \%). Al analizar los GFA considerando la biomasa, en los puntos 1 y 3 , predominó el grupo raspador y en el punto 2, el grupo colector-filtrador, dado por la presencia de un ejemplar de Mycetopodidae.

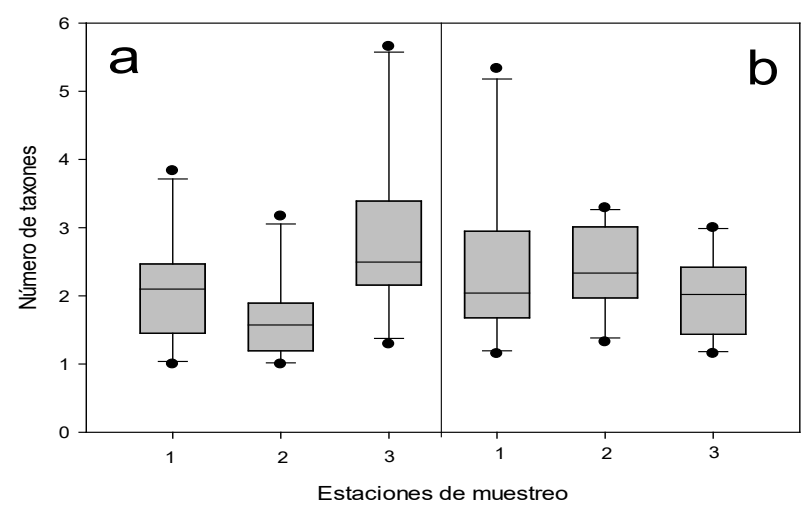

Figura 6. Gráficos de cajas discriminados por estación de muestreo: a) Número efectivo de especies (número de Hill N1). b) Número de especies dominantes (número de Hill N2).

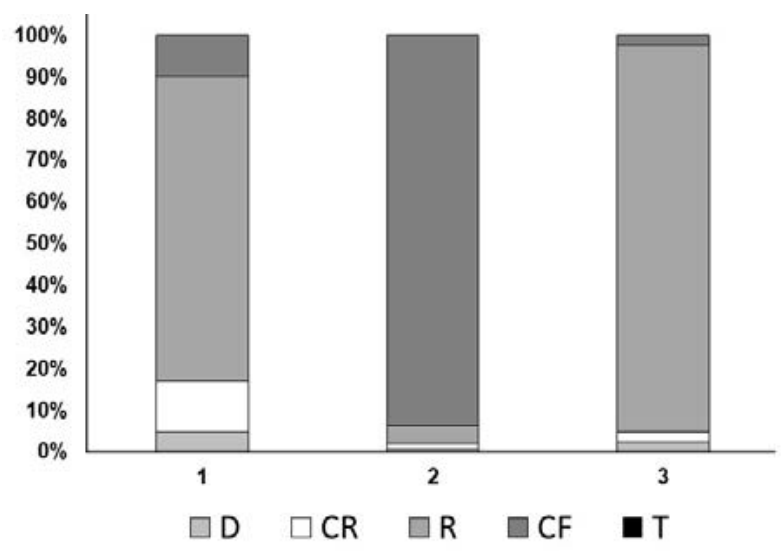

Figura 7. Biomasa de GFA (en \%) por punto de muestreo (1, 2, 3), $\mathrm{D}=$ depredador, $\mathrm{CR}=$ colector-recolector, $\mathrm{R}=$ raspador, $\mathrm{CF}=$ colector-filtrador, $\mathrm{T}=$ fragmentador. 
Finalmente, los sedimentos en el punto 1 y 3 estuvieron compuestos principalmente por materia orgánica de gran tamaño y limo. A diferencia de estos, el punto 2 presentó materia orgánica de pequeño tamaño, arena y grava.

\section{Discusión}

Este trabajo representa el primer reporte de la riqueza de macroinvertebrados bentónicos y el primer aporte sobre la estructura del ensamblaje en la laguna del Parque Unzué y un aporte al conocimiento de este tipo de ecosistema en la región pampeana. Al respecto, Rodríguez-Barrios et al. (2011) señalan la importancia de generar información que integre elementos de la estructura de las comunidades y la función de estos organismos en los ecosistemas. Al analizar los ensamblajes de invertebrados bentónicos desde una perspectiva funcional, es posible obtener información de cómo cambian en respuesta a perturbaciones o cambios en el medio (Pavé y Marchese, 2005).

Los parámetros in situ determinados en el presente estudio fueron similares a los reportados previamente para el área de estudio (Gianello, 2016) y para arroyos de la zona (Crettaz-Minaglia et al., 2014; Juárez et al., 2016; Gianello et al., 2016), excepto en la conductividad eléctrica y los SDT que fueron más bajos en la laguna que en los arroyos de la zona. Valores similares de CE se encontraron en estudios realizados en lagos artificiales de la provincia de Córdoba por Novoa et al. (2006; 2011). Estos parámetros suelen ser similares a los del río Gualeguaychú (datos no mostrados), el curso de agua que alimenta la laguna ante eventuales recambios de agua y crecidas extraordinarias. Respecto a la influencia de los aportes a la laguna, se observó incremento de las precipitaciones locales durante la primavera-verano, lo que generó anegamiento del Parque Unzué y, además, incremento el nivel hidrométrico del río Gualeguaychú en otoño-invierno. Esto último pudo haberse debido al ingreso de agua proveniente de la cuenca alta del río Uruguay (por el norte) y a efectos de la sudestada que genera ingreso de agua del río de La Plata (por el sur), afectando, en ambos casos, el nivel hidrométrico del río Gualeguaychú. Los valores de oxígeno disuelto fueron bajos ( $<5,0 \mathrm{mg} / \mathrm{L}$, condiciones de hipoxia) en el $60 \%$ de las mediciones, situación que puede generar la pérdida de taxones sensibles (Goyenola, 2007). Los mayores valores fueron registrados en primavera debido a la mayor actividad del fitoplancton. La laguna del Parque Unzué fue previamente registrado como un sistema eutrófico que presenta usualmente valores elevados de fósforo y materia orgánica (Gianello, 2016). En relación con esto, la riqueza de macroinvertebrados bentónicos fue relativamente baja y la mayoría de los taxones identificados fueron característicos de ambientes con abundante materia orgánica en sus sedimentos y bajos valores de oxígeno disuelto como Oligochaeta, Chironomidae (Alonso y Camargo, 2005), Cochliopidae (Paréda Rosa et al., 2010), Hirudinea y P. canaliculata (Rodríguez-Capítulo et al., 2001). Se detectó la presencia de la especie invasora $C$. fluminea (Darrigran y Pastorino, 1993) en la laguna del Parque Unzué; esto figura como el primer reporte en dicha área de estudio.

Las diferencias entre los puntos de muestreo con respecto a los ensamblajes de macroinvertebrados pueden deberse al tipo de sustrato dado que suele ser un aspecto de importancia ecológica (Ward, 1992). Los puntos 1 y 3 presentaron un sedimento con abundante materia orgánica gruesa como hojas y pequeñas ramas; en cambio el punto 2 presentó un sedimento con arena, pequeñas piedras y menor proporción de materia orgánica. El tipo de sustrato de la laguna también puede ser un factor atribuido a la baja riqueza, ya que tanto las plantas acuáticas (Armitage et al., 1995) como los guijarros (Marchant y Barmuta, 1994) ofrecen disponibilidad de hábitat, refugio y alimento para los macroinvertebrados. En relación con los estudios de los ensamblajes de macroinvertebrados, teniendo en cuenta los GFA, son escasos y no se han encontrado trabajos publicados para la Pampa Mesopotámica. En el presente estudio, el grupo predominante fue el CR, representado principalmente por Oligochaeta, lo cual coincide con el estudio realizado por Almeida (2015) en lagunas urbanas de la región Agreste (Paraibano, Brasil) donde los CR predominaron en todos los sitios de muestreo, seguidos de los R. Este grupo funcional ha sido frecuentemente encontrado en diversos ambientes, como arroyos (Príncipe et al., 2010), estuarios (Silva et al., 2009), ríos de llanura (Pavé y Marchese, 2005) y ríos de montaña (Miserendino y Pizzolón 2000; Velázquez y Miserendino, 2003). Los GFA se encuentran asociados a la oferta de recursos y el tipo de metabolismo del sistema, por lo tanto, podrían ser un buen criterio de indicación de la calidad de su hábitat, como complemento a los sistemas de evaluación, basados en composición y en estructura (Motta Díaz et al., 2016).

\section{Agradecimientos}

Los autores queremos agradecer a la Universidad Autónoma de Entre Ríos por el financiamiento a través del proyecto "Diagnóstico ambiental y propuesta de gestión para la Laguna del Parque Unzué (Gualeguaychú, Entre Ríos)” Res. C.S. 404/15 y la beca de iniciación a la investigación (Resolución CS 105/16). A los integrantes del Laboratorio de Indicadores Biológicos y Gestión Ambiental de Calidad de Agua (FCyT-UADER) por su colaboración 
en los muestreos.También, a los evaluadores anónimos, proporcionados por la revista, por sus importantes aportes a la mejora del presente trabajo.

\section{Referencias}

Alonso, A. y Camargo, J.A. 2005. Estado actual y perspectivas en el empleo de la comunidad de macroinvertebrados bentónicos como indicadora del estado ecológico de los ecosistemas fluviales españoles. Revista Ecosistemas 14(3): 87-99.

Almeida, R.S.R. 2015. Avaliação da estrutura trófica de macroinvertebrados bentônicos em lagoas artificiais no semiárido. Tesis de pregrado, UEPB Campina, Grande, Brasil.

Andramunio-Acero, C. y Caraballo, P. 2012. Análisis de las relaciones tróficas en un lago de inundación de la amazonia colombiana. Revista Colombiana de Ciencia Animal-RECIA 4(1): 102-120.

Armitage, P.D., Pardo, I. y Brown, A. 1995. Temporal constancy of faunal assemblages in 'mesohabitats' - Application to management? Archiv für Hydrobiologie 133:367-387.

APHA-AWWA-WFF. 2013. Standard Method for the Examination of Water and Wastewater. $17^{\circ}$. Díaz de Santos S.A. (Ed). Madrid.

Barbour, M.T., Gerritsen, J., Snyder, B.D., Stribling, J.B. y Faulkner, C. 1999. Rapid Bioassessment Protocols for Use in Streams and Wadeable Rivers: Periphyton, Benthic Macroinvertebrates, and Fish. Environmental Protection Agency, Office of Water, Washington D.C.

Bray J.R. y Curtis J.T. 1957. An ordination of the upland forest communities of southern Wisconsin. Ecological Monographs 27: 325-349.

Crettaz-Minaglia, M. C., Juárez, R., Aguer, I., Borro, E. y Peruzzo, R. 2014. Aplicación de índices de calidad de agua en un arroyo pampeano utilizando macroinvertebrados bentónicos como bioindicadores (Gualeguaychú, Entre Ríos, Argentina). Biología Acuática 30: 93 - 105.

Cummins, K. W., Merritt, R. W. y Andrade, P. C. 2005. The use of invertebrate functional groups to characterize ecosystem attributes in selected streams and rivers in south Brazil. Studies on Neotropical Fauna and Environment 40(1): 69-89.

Darrigran, G. y Pastorino, G. 1993. Bivalvos invasores en el Río de la Plata, Argentina. Comunicaciones de la Sociedad
Darrigran, G., Vilches, A., Legarralde, T. y Damborenea, C. 2007. Guía para el estudio de macroinvertebrados: I-Métodos de colecta y técnicas de fijación. ProBiota, FCNyM, UNLP. La Plata.

Domínguez, E. y Fernández, H.R. 2009. Macroinvertebrados bentónicos sudamericanos: sistemática y biología. Fundación Miguel Lillo, Tucumán.

Elosegui, A. y Sabater, S. 2009. Conceptos y técnicas en ecología fluvial. Fundación BBVA, Bilbao.

Faggi, A. y Martínez-Carretero, E. 2013. Argentina. En: Mcgregor-Fors, I. y Ortega-Álvarez, R. Editores. Ecología urbana: experiencias en América Latina. México D.F.

Ferreira Rezende, C. 2007. Estrutura da comunidade de macroinvertebrados associados ao folhiço submerso de remanso e correnteza em igarapés da Amazônia Central. Biota Neotropica 7(2): 301-306.

García-Rodríguez, J., Molina-Astudillo, F.I., MirandaEspinoza, E., Soriano-Salazar, M.B. y Díaz- Vargas, M. 2014. Variación fitoplanctónica en un lago urbano del municipio de Cuernavaca, Morelos, México. Acta Universitaria 25(1): 3-11.

Gianello, D. 2016. Diagnóstico y gestión ambiental en la Laguna del Parque Unzué (Gualeguaychú, Entre Ríos) con énfasis en el estudio de la diversidad de fitoplancton. Tesis de licenciatura. Facultad de Ciencia y Tecnología, Universidad Autónoma de Entre Ríos, Argentina.

Gianello, D., Juárez, R., Aguer, I. y Crettaz Minaglia, M.C. 2016. Riqueza de fitoplancton en el arroyo La Cañada (Gualeguaychú, Entre Ríos). Scientia Interfluvius 7(2): 29-36.

Gianello, D., Aguer, I. y Crettaz-Minaglia, M. 2017. Aportes al conocimiento del fitoplancton de la laguna del Parque Unzué (Gualeguaychú, Argentina). Natura Neotropicalis 2(48): 37-43.

Goyenola, G. 2007. Guía para la utilización de las valijas viajeras: oxígeno disuelto. Red MAPSA, versión 1.0. Montevideo, Uruguay. Disponible en: http://imasd.fcien.edu. uy/difusion/educamb/propuestas/red/curso_2007/cartillas/ tematicas/OD.pdf. Consultado: 9 de abril 2018.

Hammer, Ø., Harper, D.A.T y Ryan, P.D. 2001. PAST: Paleontological statistics software package for education and data analysis. Palaeontologia Electronica 4(1): 1-9.

Hill, M. O. 1973. Diversity and evenness: a unifying notation and its consequences. Ecology 54(2): 427-432.

Hough, J.L. 1998. Financing marine protected areas: the role of the GEF. Parks 8: 53-49. 
INDEC. 2010. Censo Nacional de Población, Hogares y Viviendas. Disponible en: http://www.entrerios.gov.ar/dec/ paginas/municipios.html. Consultado: 19 de julio de 2014.

Juárez, R., Crettaz Minaglia, M.C., Aguer, I., Juárez, I., Gianello, D., Ávila, E. y Roldán, C. 2016. Aplicación de índices bióticos de calidad de agua en cuatro arroyos de la cuenca del río Gualeguaychú (Entre Ríos, Argentina). Intropica 11: 35 -46.

Lopretto, E.C. y Tell, G. 1995. Ecosistemas de aguas continentales. Ediciones Sur, La Plata.

Mancini, M., Crichigno, S., Ortíz, M. y Haro, J.G. 2012. Lagos urbanos: Importancia, dinamismo y multiplicidad de usos. El caso del lago Villa Dalcar. Biología Acuática 27: 175-189.

Marchant, R. y Barmutta, L. 1994. The ordination of macroinvertebrate communities from stream in Victoria, Australia. Verhandlungen des Internationalen Verein Limnologie 25: 1835-1836.

Merritt, R.W. y Cummins, K.W. 1978. An Introduction to the Aquatic Insects of North America. Edition. Kendall Hunt Pub. Co.

Miserendino, L.M., y Pizzolón, L.A. 2000. Macroinvertebrates of a fluvial system in Patagonia: altitudinal zonation and functional structure. Archiv für Hydrobiologie 55-83.

Montoya, J.V., Castillo, M.M. y Sánchez, L. 2011. La importancia de las inundaciones periódicas para el funcionamiento y conservación de los ecosistemas inundables de grandes ríos tropicales: estudios en la cuenca del Orinoco. Interciencia 36(12): 900-907.

Morello, J., Matteucci, S.D., Rodríguez, A.F. y Silva, M.E. 2012. Ecorregiones y complejos Ecosistémicos de Argentina. Orientación Gráfica Editora, Buenos Aires.

Motta Díaz, Á., Ortega Corredor, L., Niño Fernández, Y. y Aranguren Riaño, N. 2016. Grupos funcionales alimenticios de macroinvertebrados acuáticos en un arroyo tropical (Colombia). Revista UDCA Actualidad \& Divulgación Científica 19(2): 425-433.

Novoa, M., Luque, M.E., Lombardo, D. y Martínez de Fabricious, A. L. 2006. Estudio ficológico de lagos urbanos artificiales del sur de la provincial de Córdoba. Boletín Sociedad Argentina Botánica 41(3-4): 203-231.

Novoa, M., Martínez de Fabricius, A.L., Luque, M.E. y M. Lombardo, D. 2011. Distribución temporal del fitoplancton en un lago urbano del centro de Argentina (Río Cuarto, Córdoba). Biológicas 13(2): 1-14.
Ocon, C., Oosterom, M.V.L.V., Muñoz, M.I. y RodriguesCapítulo, A. 2013. Macroinvertebrate trophic responses to nutrient addition in a temperate stream in South America. Fundamental and Applied Limnology/Archiv für Hydrobiologie 182(1) 17-30.

Ospina A. N. y Peña, J. E. 2004. Alternativas de Monitoreo de Calidad de Aguas: Algas como Bioindicadores. Acta Nova 2(4): 514-517.

Paré da Rosa, L.P., Querol, E. y Koenemann, J.G. 2010. Aspectos ecológicos de Cochliopidae y Lithoglyphidae (Mollusca, Gastropoda) do arroio Felizardo, Bacia do río Uruguay medio, Pampa Brasileiro (Dados Parciais). Biodiversidade Pampeaana 8(1): 7-13.

Pave, P.J. y Marchese, M. 2005. Invertebrados bentónicos como indicadores de calidad del agua en ríos urbanos (ParanáEntre Ríos, Argentina). Ecología austral 15(2): 183-197.

Pla, L. 2006. Biodiversidad: Inferencia basada en el índice de Shannon y la riqueza. Interciencia 31(8): 583-590.

Plafkin, J.L., Barbour, M., Porter, K.D., Gross, S.K. y Hughes, R.M. 1989. Rapid bioassessment protocols for use in streams and rivers: Benthic macroinvertebrates and fish. EPA. Washington D.C.

Prat, N., Ríos, B., Acosta R. y Rieradevall, M. 2009. Los macroinvertebrados como indicadores de calidad de las aguas. En: Domínguez, E. y Fernández, H. Editores. Macroinvertebrados bentónicos sudamericanos. Sistemática y biología. Fundación Miguel Lillo, San Miguel de Tucumán.

Príncipe, R.E., Gualdoni, C.M., Oberto, A.M., Raffaini, G.B. y Corigliano, M.C. 2010. Spatial-temporal patterns of functional feeding groups in mountain streams of Córdoba, Argentina. Ecología Austral 20: 257-268.

Quirós, R. 2007. Manejo y recuperación de lagos urbanos. Universidad de Buenos Aires, Buenos Aires.

Rodríguez-Barrios, J., Ospina-Tórres, R. y Turizo-Correa, R. 2011. Grupos funcionales alimentarios de macroinvertebrados acuáticos en el río Gaira, Colombia. Revista de Biología Tropical 59(4): 1537-1552.

Rodrígues-Capítulo, A., Tangorra, M. y Ocón, C. 2001. Use of benthic macroinvertebrates to assess the biologist status of pampean streams in Argentina. Aquatic Ecology 35:109-119.

Rodrígues-Capítulo, A., Gómez, N., Giorgi, A. y Feijoó, C. 2010. Global changes in Pampean lowland streams (Argentina): implications for biodiversity and functioning. Hydrobiología 657: 53-70. 
Sierra-Vásquez, M.A. 2012. Ciudad y fauna urbana: un estudio de caso orientado al reconocimiento de la relación hombre, fauna y hábitat urbano en Medellín. Tesis Doctoral. Universidad Nacional de Colombia, Sede Medellín, Colombia.

Shannon, C.E. y Weaver, W. 1949. The Mathematical Theory of Communication. Urbana, IL: University of Illinois Press, Illionis.

Silva, F.L. Da., Pauleto, G. M., Talamoni, J.L.B. y Ruiz, S.S. 2009. Categorização funcional trófica das comunidades de macroinvertebrados de dois reservatórios na região Centro - Oeste do Estado de São Paulo, Brasil. Acta Scientiarum Biological Sciences 31(1): 73-78.

Sorensen, M., Barzetti, V., Keipi K. y Williams, J. 1998. Manejo de las áreas verdes urbanas. Documento de buenas prácticas. División de Medio Ambiente del Departamento de Desarrollo Sostenible del Banco Interamericano de Desarrollo, Washington D.C.
Taborda, V., Gianello, D., Aguer, I. y Crettaz Minaglia, M. 2017. Importancia de la conservación de las lagunas urbanas pampeanas. Congreso Internacional Aguas, Ambiente y Energía, Mendoza, Argentina. Acta de congreso 346-350.

Velásquez, S.M. y Miserendino, M.L. 2003. Análisis de la materia orgánica alóctona y organización funcional de macroinvertebrados en relación con el tipo de hábitat en ríos de montaña de Patagonia. Ecología austral 13(1): 67-82.

Villanueva, M., y Chanamé Zapata, F. C. 2016. Análisis de la biodiversidad de macroinvertebrados bentónicos del río Cunas mediante indicadores ambientales, Junín-Perú. Scientia Agropecuaria 7(1): 33-44.

Ward, J.V. 1992. Aquatic insect ecology. John Wiley \& Sons. New York.

Citar como: Crettaz-Minaglia M., Gianello, D., Roldán, C., Chaves, E., Aguer I. y Juárez, R. 2018. Estructura del ensamblaje de macroinvertebrados bentónicos de una laguna urbana de la Pampa Mesopotámica. Intropica 13(2):112-121. DOI: http://dx.doi.org/ 10.21676/23897864.2557. 